\title{
Single Tilt Method for Residual Stress Evaluation with 2D Detectors
}

\author{
Bob B. He \\ Bruker AXS Inc, 5465 East Cheryl Parkway, Madison, WI 53711, USA \\ bob.he@bruker.com
}

Keywords: 2D detector, Residual stress, Coating, Thin films, Polymer.

\begin{abstract}
When X-ray diffraction is used for residual stress measurement, high $2 \theta$ peaks are typically used for enhanced $2 \theta$ shift and better tolerance to the sample height error. But for thin films, coatings, or polymer materials, high $2 \theta$ peaks may not be available or appropriate for stress measurement. As a result of large angular coverage with a 2D detector, residual stress can be measured with a single tilt angle. The diffraction vector coverage from low $2 \theta$ angle diffraction ring can satisfy the stress or stress tensor measurement at a single tilt angle. The single tilt method can avoid the sample height error caused by changing the tilt angle, which is especially critical when measuring stress with a low $2 \theta$ peak. Another advantage is the consistent depth of penetration due to a constant incident angle, which is especially suitable for residual stress measurement on coatings, thin films or samples with steep stress gradient. This paper introduces the single tilt method for stress evaluation with two-dimensional detectors, including experimental examples on coatings and polymers.
\end{abstract}

\section{Introduction}

Measurement of residual stresses in thin films or coatings by X-ray diffraction is always a challenge due to weak diffraction signals from the thin layer, sharp stress or strain gradients, preferred orientation, anisotropic grain shape and inhomogeneous phase and microstructure distribution [1]. When residual stresses are measured on a coating or thin film sample, it is preferable to keep a small incident angle or control the incident angle to get the most X-ray scattering from the thin film layer. Generally, high $2 \theta$ peaks are preferred for stress measurement due to the more significant $2 \theta$ shift and less sensitive to the sample height error. But for thin films, coatings, or polymer materials, high $2 \theta$ peaks may not be available or appropriate for stress measurement. With low $2 \theta$ peaks, it is more difficult or even impossible to measure stress with the conventional $\sin ^{2} \psi$ method. With iso-inclination method, the incident angle varies during data collection so the incident angle cannot be kept low during data collection, while with side-inclination method, the actual incident angle to the sample surface is further reduced, and the measurement results become extremely sensitive to the sample height error.

With two-dimensional X-ray diffraction $\left(\mathrm{XRD}^{2}\right)$, stress measurement is based on a direct relationship between stress tensor and diffraction cone distortion [2]. For a diffraction ring with low $2 \theta$, the diffraction vectors cover more directions at each measurement so that sufficient angular coverage can be achieved with a single tilt. Therefore, the data collection can be done at a fixed $\psi$ angle with only $\phi$ rotation. Since the incident angle is constant, the depth of penetration as a function of $\gamma$ is consistent at all $\phi$ angles. For most goniometer with Eulerian geometry, the $\phi$ axis is typically built on precision bearing with very small spherical error, while $\psi$ rotation is achieved by a circular track which tends to have much more significant spherical error. Avoiding $\psi$ rotation can significantly reduce the sample height variation during the data collection, 
therefore improve the measurement accuracy. As long as the sample height is consistent at various $\phi$ angles, deviation of the sample surface from the instrument center may affect the pseudo-hydrostatic stress, but has minimal effect on the stress results.

\section{Geometry and $\Delta \psi$ Coverage}

Figure 1 illustrates the diffraction vector distribution for the diffraction pattern collected with a point (0D) detector and a 2D detector. The hemisphere represents all the possible orientations from the origin $\mathrm{O}$ of the sample coordinate $\mathrm{S}_{1} \mathrm{~S}_{2} \mathrm{~S}_{3}$. With a point detector, at $\psi=0^{\circ}$, the diffraction vector points to the sample normal direction $S_{3}$. In order to measure stress, the sample has to be tilted at several $\psi$ angles, for instance $0^{\circ}, 15^{\circ}, 30^{\circ}$ and $45^{\circ}$ as indicated by the purple mark $\otimes$. With a 2D detector, the trace of the diffraction vector covers a range as shown by the red curve. The diffraction vectors $H_{1}$ and $H_{2}$ correspond to the two extreme values of $\gamma_{1}$ and $\gamma_{2}$ on the diffraction ring covered by the 2D detector, $\Delta \psi$ is the total angular range of the diffraction vector distribution, and $\Delta \gamma$ is the $\gamma$ range. At a given tilt angle $\psi$, for example $22.5^{\circ}$, the diffraction vector covers a range as shown by the green curve. For low $2 \theta$ diffraction rings at proper detector distance, it is possible to cover sufficient angular range for stress evaluation with a single tilt. The complete data set for stress tensor can be collected at several $\phi$ angles, for instance $360^{\circ}$ scan with $45^{\circ}$ steps. Therefore the complete data set are collected with $\phi$ scan only.

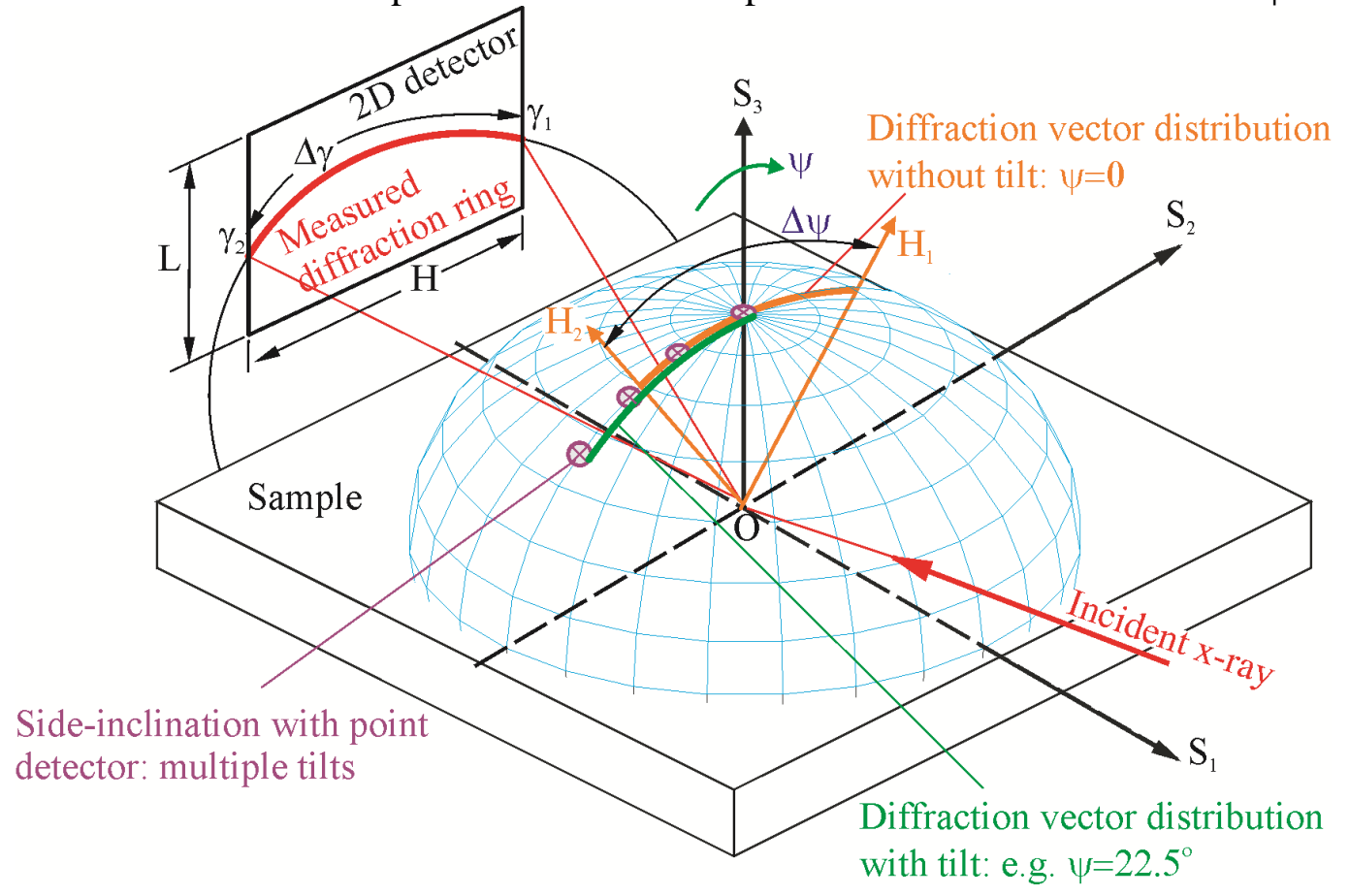

Figure 1. Diffraction vector distribution for $O D$ and $2 D$ detectors.

The diffraction vector distribution range $(\Delta \psi)$ is determined by the detector distance $\mathrm{D}$, detector height $\mathrm{H}$, detector width $\mathrm{L}, 2 \theta$ angle and detector swing angle $\alpha$. Calculating the $\Delta \psi$ coverage for a flat detector involves solving some implicit equations [2]. For simplicity, an equation derived from cylindrical detector can be used for $\Delta \psi$ calculation with negligible error:

$$
\Delta \psi=2 \arcsin \left(\frac{H}{2 \sin \theta \sqrt{4 D^{2}+H^{2}}}\right)
$$


Here we assume the $\gamma$ range is limited by the detector height $\mathrm{H}$. The measured $\gamma$ range may also be limited by the detector width $\mathrm{L}$ when $\mathrm{L}$ is too small or the detector swing angle is not properly set. Figure 2 shows the diffraction vector distribution range $\Delta \psi$ as a function of $2 \theta$ calculated for EIGER 2R $500 \mathrm{k}^{\mathrm{TM}}$ detector at various detector distance and in $\gamma$-optimized orientation $(\mathrm{H}=77.2 \mathrm{~mm}$ and $\mathrm{L}=38.6 \mathrm{~mm})$. For stress measurement with single tilt, $\Delta \psi$ of more than $30^{\circ}$ is acceptable, but the desired $\Delta \psi$ coverage is $45^{\circ}$ or above. A shorter detector distance can be used to increase $\Delta \psi$. In general, the angular coverage $\Delta \psi$ is significantly higher with low $2 \theta$ angles. Therefore, the single tilt method is more suitable for middle or low $2 \theta$ angles.

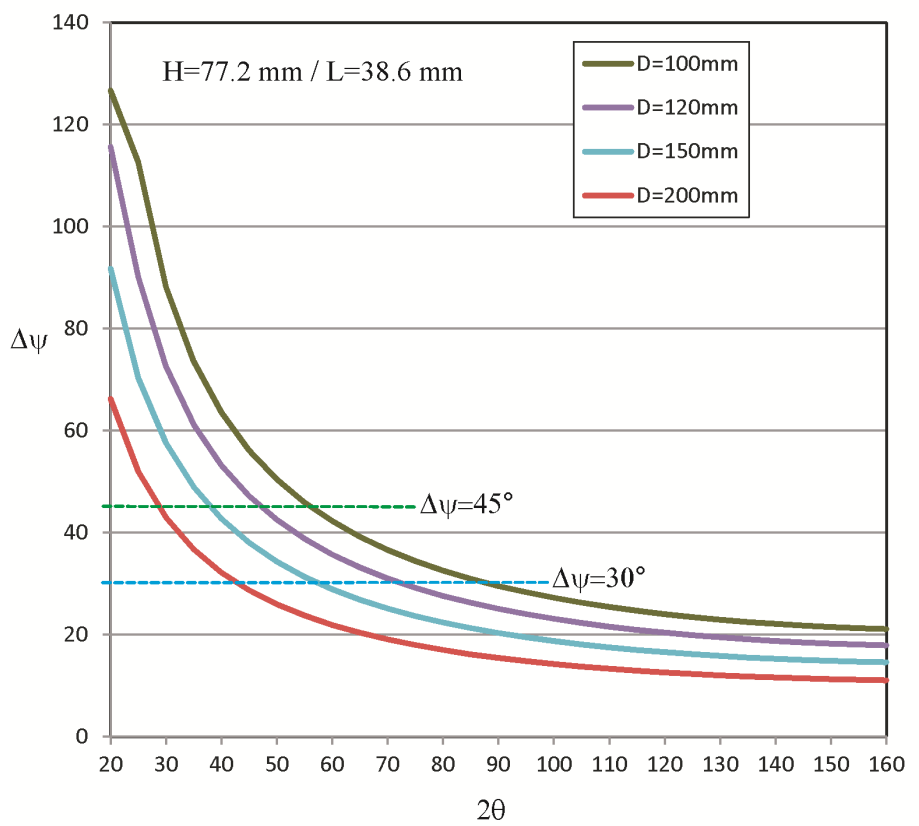

Figure 2. $\Delta \psi$ range as a function of $2 \theta$ for EIGER $2 R 500 k^{T M}$ detector.
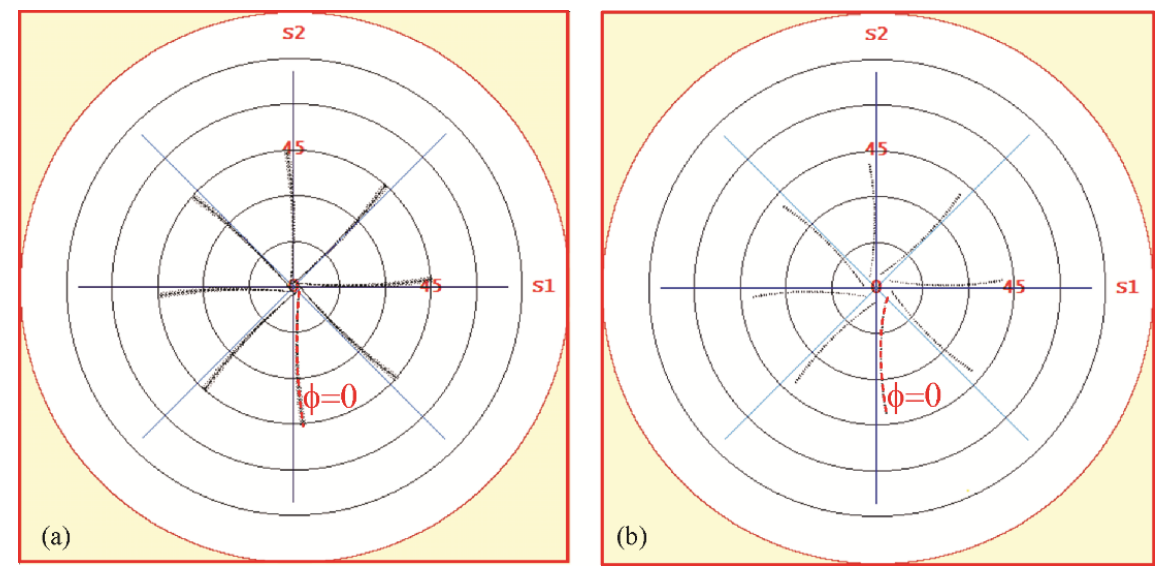

Figure 3. Data collection strategy schemes with single tilt at $\psi=22.5^{\circ}$ and complete $\phi$ rotation of $45^{\circ}$ steps. (a): $\mathrm{PE}$ polymer (020), $2 \theta=36.3^{\circ}$ and $\mathrm{D}=20 \mathrm{~cm}$; (b): $\mathrm{Al}_{2} \mathrm{O}_{3}(116), 2 \theta=57.5^{\circ}$ and $D=15 \mathrm{~cm}$.

Figure 3 illustrates the single tilt scheme generated with GADDS software for VÅTEC-500 $2 \mathrm{D}$ detector. The left (a) is for PE polymer (020) with $2 \theta=36.3^{\circ}, \psi=22.5^{\circ}$ and detector to sample 
distance $\mathrm{D}=20 \mathrm{~cm}$ and the right (b) is for $\mathrm{Al}_{2} \mathrm{O}_{3}(116)$ with $2 \theta=57.5^{\circ}, \psi=22.5^{\circ}$ and $\mathrm{D}=15 \mathrm{~cm}$. The arcs represent the trace of the diffraction vector corresponding to the data set. $S_{1}$ and $S_{2}$ are two sample orientations. The red broken curve marks the diffraction vector distribution covered by the frame collected at $\phi=0$. With eight frames collected with $\phi$ scan at $45^{\circ}$ steps, the scheme produces comprehensive orientation coverage in a symmetric distribution. The data set collected with this strategy can be used to calculate the complete biaxial stress tensor components.

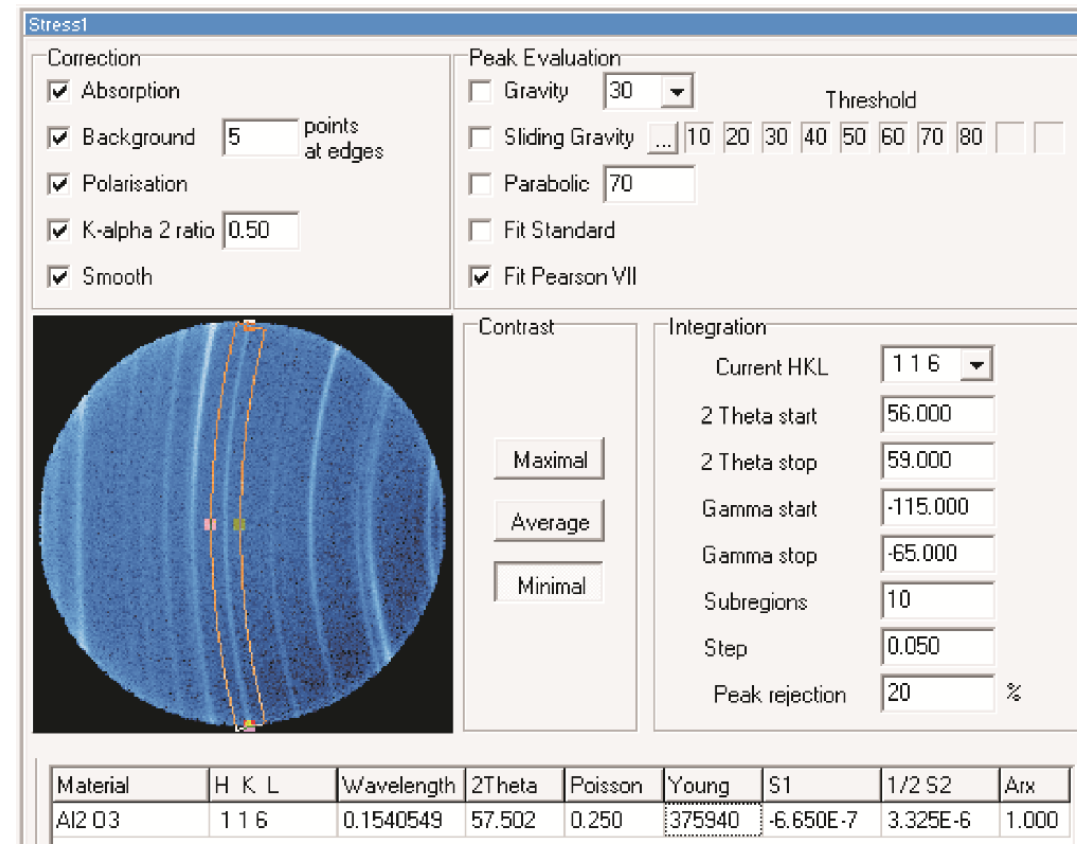

Figure 4. Data evaluation setting for $1 \mu \mathrm{m}$ thick $\mathrm{Al}_{2} \mathrm{O}_{3}$ coating on cutting insert.

The residual stress in the $\mathrm{Al}_{2} \mathrm{O}_{3}$ coating of less than $1 \mu \mathrm{m}$ thick on a proprietary cutting insert is measured with a Bruker D8-DISCOVER system containing centric Eulerian cradle and VÅNTEC-500 2D detector. With Cu-Ko radiation, the diffraction ring from (116) planes at $2 \theta=57.5^{\circ}$ is used for stress evaluation. The stress calculation is done with Bruker DIFFRAC.LEPTOS software version 7.9. Figure 4 shows the data evaluation setting. The data integration region is defined by $2 \theta$ range of $56^{\circ}$ to $59^{\circ}$ and $\gamma$ range of $-65^{\circ}$ to $-115^{\circ}$. The $50^{\circ} \gamma$ range is divided into 10 subregions, $5^{\circ}$ for each subregion. The counts within each subregion are integrated into a diffraction profile and the $2 \theta$ peak position is determined by one of the five peak evaluation algorithms. In this experiment, Pearson VII function is used to fit the profile and evaluate the $2 \theta$ peak position.

Figure 5 shows the stress evaluation results from one of the data set. The charts above " $\mathrm{A}$ " are the fitted data points on $2 \mathrm{D}$ frames. The charts above " $\mathrm{B}$ " are fitted data points in $\gamma-2 \theta$ rectangular coordinates with magnified $2 \theta$ scale, in which, black line indicates $2 \theta_{0}$, blue cross and line indicates the data points from the profile fitting of each subregion, and red line represents the calculated diffraction rings from the stress results. The scattering of the crosses about the red line represents the quality of the data, affecting the standard deviation of the stress results. Any roll error of the detector will change the trend of the fitted data points and the red line, thus the stress results. By click on any data point, the integrated profile displays above " $C$ ". With 60 seconds per frames, the total data collection time is 8 minutes. The measured stress 
values are given in the region " $\mathrm{D}$ " as $\sigma_{11}=954.7 \mathrm{MPa}, \sigma_{22}=957.9 \mathrm{MPa}$ and standard deviation 26.5MPa $(<3 \%)$.

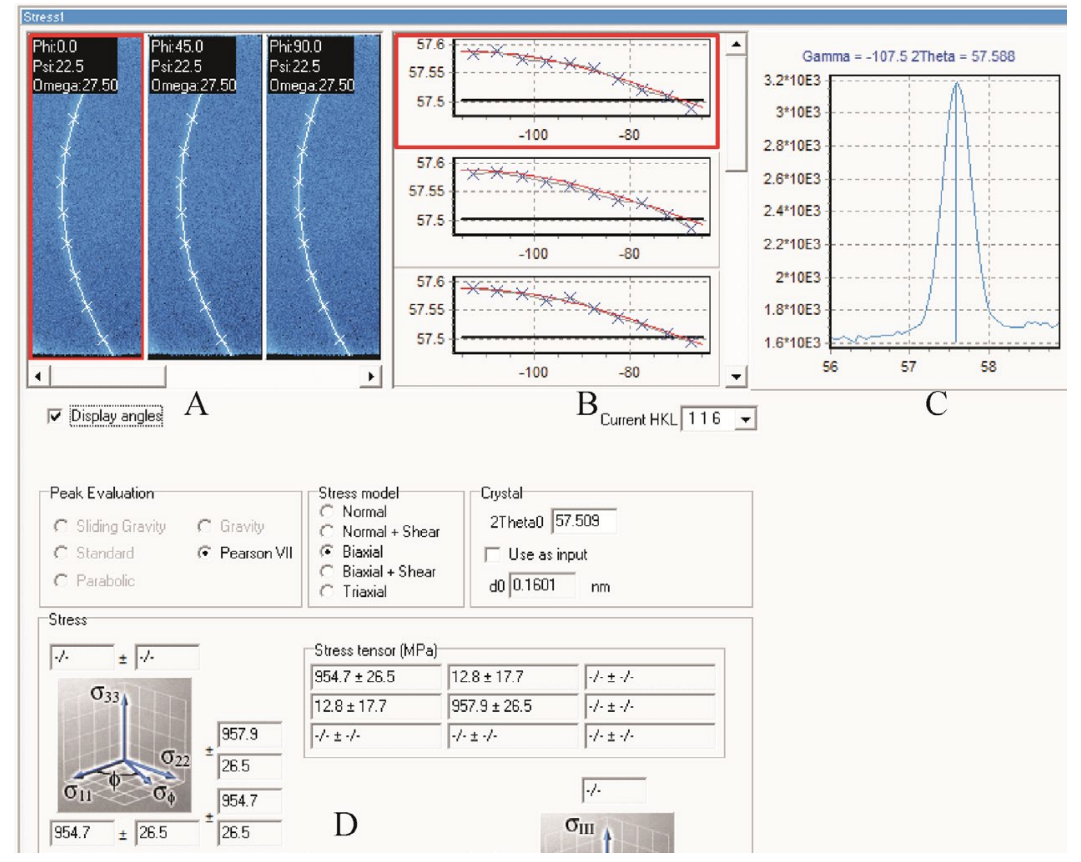

Figure 5. Data evaluation results for $1 \mu \mathrm{m}$ thick $\mathrm{Al}_{2} \mathrm{O}_{3}$ coating on cutting insert.

The single tilt stress measurement method is especially suitable for measuring residual or loading stresses in polymers. For stress measurement by X-ray diffraction, the polymer sample must contain sufficient crystalline phase. The crystalline peaks from polymers are typically with low $2 \theta$ angles. For instance, polyethylene (PE) polymer contains mainly three diffraction rings at $2 \theta$ about $21.4^{\circ}, 23.6^{\circ}$ and $36.3^{\circ}$ with $\mathrm{Cu}-\mathrm{K} \alpha$ radiation, corresponding to crystalline planes of (110), (200) and (020) respectively. Even with the $36.3^{\circ}$ peak, a large error in stress result can be introduced by the sphere of confusion with multiple sample tilt angles. The single tilt method can overcome this problem. Other challenge for stress measurement on polymers is the low stress value. However, due to the extremely low Young's modulus, such as $1070 \mathrm{MPa}$ for high density polyethylene, the $2 \theta$ shift (strain) is also more significant for the same stress if compared with metals. Due to large depth of penetration, the zero normal stress assumption $\left(\sigma_{33}=0\right.$ on sample surface) for polymers may not be as accurate as for metals.

Residual stresses on a high-density polyethylene (HDPE) pipe are measured with the single tilt method [3]. An $\mathrm{XRD}^{2}$ system in vertical $\theta-\theta$ configuration (Bruker D8-DISCOVER ${ }^{\mathrm{TM}}$ ) with $\mathrm{I} \mu \mathrm{S}^{\mathrm{TM}} \mathrm{Cu}$ microsource and VANTEC-500 ${ }^{\mathrm{TM}} 2 \mathrm{D}$ detector is used for the measurement. The HDPE pipe has a diameter of $32 \mathrm{~mm}$ and wall thickness of $3 \mathrm{~mm}$. The length of the sample is 50 $\mathrm{mm}$, cut from a pipe. Residual stresses of a total 7 points in the outer surface of the pipe and along the axial direction were measured. The depth of penetration corresponding to $50 \%$ of the total diffracted intensity is $0.3 \mathrm{~mm}$ estimated from the diffraction condition in diffractometer plane $\left(\gamma=-90^{\circ}\right)$. To avoid relaxation effect near the cutting edges, the measurement starts at 10 $\mathrm{mm}$ from one end with $5 \mathrm{~mm}$ steps and completes at the last point $10 \mathrm{~mm}$ from the other end. A total of 8 frames are collected for each measurement point with the data collection strategy given in Figure 3(b). Figure 6 shows the data evaluation setting and fitting results with LEPTOS software for one of the measurement point. The measured stresses are tensile in the pipe 
extrusion direction with variation of $1.3 \sim 2.2 \mathrm{MPa}$ over the 7 points, and compressive in the hoop direction with variation of $6.5 \sim 8.1 \mathrm{MPa}$.

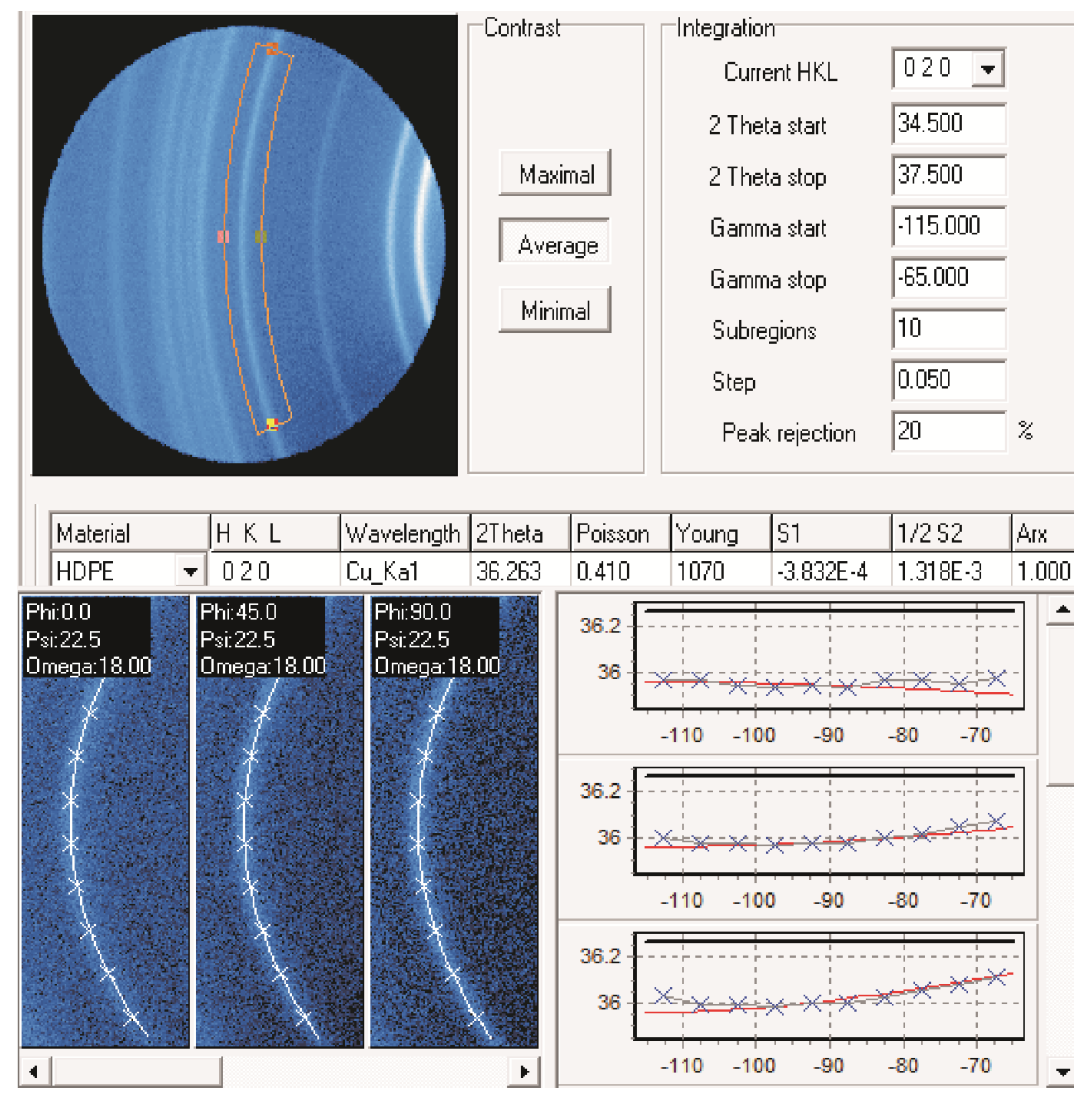

Figure 6. Data evaluation setting and fitting results for the HDPE pipe.

\section{Summary}

For thin films, coatings, or polymers, when the diffraction peaks at high $2 \theta$ angles are not available or appropriate, a low $2 \theta$ peak may be used for stress evaluation. With diffraction rings at low $2 \theta$ angle, the diffraction vector distribution can satisfy the angular coverage for stress or stress tensor measurement at a fixed tilt angle $(\psi)$. Without $\psi$ change during data collection and with $\phi$ rotation only, the sample height is accurately maintained and data collection time is reduced. The single tilt method is a unique method only achievable with an $\mathrm{XRD}^{2}$ system, which can measure residual stress with high accuracy and high speed for thin films, coatings and polymers with low to medium $2 \theta$ peaks.

\section{References}

[1] B. B. He, Measurement of residual stresses in thin films by two-dimensional XRD, Materials Science Forum, Vols. 524-525, (2006) 613-618.

https://doi.org/10.4028/www.scientific.net/MSF.524-525.613

[2] B. B. He, Two-dimensional X-ray Diffraction, $2^{\text {nd }}$ ed., John Wiley \& Sons, 2018. https://doi.org/10.1002/9781119356080

[3] M. Ren, C. Zheng, Y. Shi, Y. Tang, B. He, Residual stress measurement of high-density polyethylene pipe with two-dimensional X-ray diffraction, Adv. X-ray Anal. 61 (2018). 